\title{
6-PACK programme to decrease fall injuries in acute hospitals: cluster randomised controlled trial
}

\author{
Anna L Barker,, Renata T Morello, ${ }^{1}$ Rory Wolfe, ${ }^{1}$ Caroline A Brand, ${ }^{1}$ Terry P Haines, ${ }^{2}$ Keith D Hill, ${ }^{3}$ \\ Sandra G Brauer, ${ }^{4}$ Mari Botti, ${ }^{5}$ Robert G Cumming, ${ }^{6}$ Patricia M Livingston, ${ }^{5}$ Catherine Sherrington, ${ }^{7}$ \\ Silva Zavarsek, ${ }^{8}$ Richard I Lindley, ${ }^{7}$ Jeannette Kamar ${ }^{9}$
}

${ }^{1}$ Department of Epidemiology and Preventive Medicine,

Monash University, Melbourne, VIC 3004, Australia

2Physiotherapy Department, Monash University, Allied Health Research Unit, Monash Health, Kingston Centre, Cheltenham, VIC 3195, Australia

${ }^{3}$ School of Physiotherapy and

Exercise Science, Curtin University,

Perth, WA 6102, Australia

${ }^{4}$ Division of Physiotherapy,

School of Health and

Rehabilitation Sciences,

University of Queensland,

Brisbane, QLD 4072, Australia

${ }^{5}$ School of Nursing and

Midwifery, Deakin University,

Burwood, VIC 3125, Australia

${ }^{6}$ School of Public Health,

University of Sydney, Sydney, NSW 2006, Australia

${ }^{7}$ George Institute for Global Health, Sydney Medical School, University of Sydney, Sydney, NSW 2006, Australia

${ }^{8}$ Centre for Health Economics, Monash Business School,

Monash University, Clayton, VIC 3800, Australia

${ }^{9}$ Northern Hospital, Northern Health, Epping, VIC 3076, Australia

Correspondence to: A Barker anna.barker@monash.edu

Additional material is published online only. To view please visit the journal online (http://dx.doi. org/10.1136/bmj.h6781)

Cite this as: BMJ 2016;352:h6781 http://dx.doi.org/10.1136/bmj.h6781 Accepted: 03 December 2015

\section{ABSTRACT}

OBJECTIVE

To evaluate the effect of the 6-PACK programme on falls and fall injuries in acute wards.

\section{DESIGN}

Cluster randomised controlled trial.

SETTING

Six Australian hospitals.

PARTICIPANTS

All patients admitted to 24 acute wards during the trial period.

\section{INTERVENTIONS}

Participating wards were randomly assigned to receive either the nurse led 6-PACK programme or usual care over 12 months. The 6-PACK programme included a fall risk tool and individualised use of one or more of six interventions: "falls alert" sign, supervision of patients in the bathroom, ensuring patients' walking aids are within reach, a toileting regimen, use of a low-low bed, and use of a bed/chair alarm.

\section{MAIN OUTCOME MEASURES}

The co-primary outcomes were falls and fall injuries per 1000 occupied bed days.

\section{RESULTS}

During the trial, 46245 admissions to 16 medical and eight surgical wards occurred. As many people were admitted more than once, this represented 31411 individual patients. Patients' characteristics and length of stay were similar for intervention and control wards. Use of 6-PACK programme components was higher on intervention wards than on control wards (incidence rate ratio 3.05, 95\% confidence interval 2.14 to 4.34; $\mathrm{P}<0.001)$. In all, 1831 falls and 613 fall injuries occurred, and the rates of falls (incidence rate ratio

\section{WHAT IS ALREADY KNOWN ON THIS TOPIC}

Patients falling remains a common cause of harm in acute hospitals worldwide

Recent epidemiological studies provide evidence that the harm from in-hospital

falls is increasing

Systematic reviews show that limited high quality evidence from randomised trials or observational studies exists for effective falls prevention programmes in acute hospitals

\section{WHAT THIS STUDY ADDS}

Introduction of the 6-PACK programme improved fall risk tool completion and use of fall prevention interventions recommended by best practice guidelines but had no effect on falls or fall injuries compared with usual care

The "opportunity costs" of delivering falls prevention interventions known to be ineffective are considerable, and disinvestment in these practices should be considered System level and/or environmental interventions may offer improved potency and effectiveness and should now be the focus of further investigation

$1.04,0.78$ to $1.37 ; P=0.796)$ and fall injuries $(0.96,0.72$ to 1.27; $\mathrm{P}=0.766)$ were similar in intervention and control wards.

\section{CONCLUSIONS}

Positive changes in falls prevention practice occurred following the introduction of the 6-PACK programme. However, no difference was seen in falls or fall injuries between groups. High quality evidence showing the effectiveness of falls prevention interventions in acute wards remains absent. Novel solutions to the problem of in-hospital falls are urgently needed.

TRIAL REGISTRATION

Australian New Zealand Clinical Trials Registry ACTRN12611000332921.

\section{Introduction}

Falls remain a common cause of harm to patients in acute hospitals worldwide. In the United Kingdom, as many as 250000 falls and more than 1000 fractures are recorded each year. ${ }^{1}$ Recent epidemiological studies provide evidence that the harm from in-hospital falls is increasing. A Danish study that included national hospital data showed that the rate of fall related major injuries increased more than 11\% between 2007 and 2012. ${ }^{2}$ The operational costs for fallers who are seriously injured during their hospital stay are higher than for non-fallers, and their length of stay is more than six days longer. ${ }^{34}$ Consequently, best practice guidelines on falls prevention have been developed, ${ }^{5-7}$ and in-hospital falls have been adopted as a quality indicator. ${ }^{89}$ In the United Kingdom, falls are reported under the NHS Patient Safety Thermometer ${ }^{10}$-a local improvement tool for measuring, monitoring, and analysing harms to patients and "harm free" care. A financial incentive to prevent falls exists in the United States, as hospitals do not receive reimbursement for management of serious injuries resulting from inpatient falls. ${ }^{11}$ A recent report on data from 1381 US hospitals showed no significant change in injurious falls following the implementation of the non-payment initiative. ${ }^{12}$ This is in contrast to rates of other "never events" such as hospital acquired infections, for which significant declines were reported..$^{12}$ Discussion of reasons for the reduction in hospital acquired infections but not falls has highlighted the fact that more robust evidence on how to prevent infections now exists. ${ }^{13}$

Limited high quality evidence exists for effective falls prevention programmes in acute hospitals. The most recent Cochrane systematic review concluded that, despite several trials, limited evidence supports any one intervention and that more trials are needed to confirm the effectiveness of multifactorial interventions in the 
hospital setting. ${ }^{14}$ Some evidence exists for effectiveness of prevention interventions in sub-acute or rehabilitation hospitals or in samples with an average length of stay longer than 10 days. ${ }^{15-17}$ A review that included observational studies and randomised controlled trials augments the Cochrane review and also reported a lack of evidence for reducing falls in hospital populations with a shorter length of stay, noting that successful trials had been in samples with a mean length of stay of 19 days or more. ${ }^{18}$

In many Western models of healthcare, acute hospitals provide care for short periods (average length of stay less than three days). ${ }^{19}$ Once patients are medically stable, they are discharged to either home, rehabilitation, or sub-acute care (average length of stay more than 17 days). ${ }^{19}$ The case mix also differs across settings; $48 \%$ of patients in acute hospitals are aged 65 and over compared with more than $70 \%$ in sub-acute settings. Sub-acute hospitals also have a greater number of allied health professionals and geriatricians than do acute hospitals, reflecting the rehabilitative nature of the care. These differences may partially explain why programmes have been less effective in acute hospitals than in sub-acute hospitals.

The 6-PACK programme is a targeted, nurse led, multifactorial falls prevention programme designed specifically for acute hospitals. It includes a nine item fall risk tool and six interventions. ${ }^{20}$ The components of the 6-PACK programme were selected on the basis of best available evidence at the time of development, expert opinion, and best practice guideline recommendations. Alert signs aimed to increase awareness by staff of patients at high risk of falling. Bathroom supervision and toileting regimens were included, as many falls occur in relation to toileting and these falls are also more likely to result in injury. ${ }^{2122} \mathrm{~A}$ review paper also identified toileting as one of the interventions commonly included in previous successful studies testing multifactorial programmes. ${ }^{18}$ Positioning patients' walking aids within reach was intended to improve safety of mobility, as impaired mobility is a common risk factor for falls. ${ }^{21}$ Bed/chair alarms were included to reduce the likelihood of patients with cognitive impairment moving from a bed or chair without staff assistance. Low-low beds were believed to be an effective strategy for reducing the likelihood of injury in patients with cognitive impairment who may attempt to get out of bed independently. ${ }^{23}$

Although components of the 6-PACK programme are commonplace in some hospitals, their implementation has often been suboptimal. ${ }^{24}$ The 6-PACK programme focuses on only a small number of interventions: provision of equipment and integration of documentation into existing care plans. This was intended to increase the frequency of use of the fall risk tool and six interventions. A nine year observational study involving 271095 patients from one hospital indicated that fall injuries reduced following the implementation of the 6-PACK programme. ${ }^{25}$ The apparent success of the programme may be due to the interventions not depending on multidisciplinary input. Nurses are the primary care providers for hospital patients, so they are optimally positioned to implement falls prevention activities. Having a single discipline responsible for falls prevention activities may improve accountability for implementation. The FallSafe programme is another multifactorial nurse led falls prevention programme developed for acute wards. The programme includes nine assessment and intervention components as a care bundle. The interventions included in FallSafe are different from those included in 6-PACK. A non-randomised controlled evaluation of FallSafe involving 16 hospitals in the United Kingdom reported a reduction in falls in the wards that implemented FallSafe and no change in the control wards. ${ }^{26}$

These observational studies provide proof of concept for the effectiveness of nurse led multifactorial programmes in acute wards, but large scale randomised trials are needed to provide robust estimates of effect and evidence of generalisability. We therefore did a cluster randomised controlled trial to evaluate the effect of the 6-PACK programme on falls and fall injuries in acute wards. We used a ward based approach to reduce contamination between groups. Randomisation at the ward level was appropriate, as key aspects of the intervention were ward based.

\section{Methods}

\section{Design and setting}

This cluster randomised controlled trial recruited 24 acute wards from six Australian hospitals. Wards were eligible to participate if they were nominated by participating hospitals as being wards where falls commonly occurred, had an average length of stay of patients of less than 10 days, had one or fewer low-low beds to each six standard beds on medical wards and one or fewer lowlow beds to each 29 standard beds on surgical wards, and did not include a fall risk tool or intervention checklist on the daily patient care plan documentation. Within hospitals, wards of the same type (surgical or medical) were recruited in pairs. A waiver of individual patient consent was provided by hospital ethics committees, enabling all patients on participating wards to be included as trial participants. There were no patient level exclusion criteria. A detailed description of the study methods is available in the published protocol and trial registration (www.anzctr.org.au number ACTRN12611000332921). ${ }^{27}$

\section{Intervention}

The intervention, the 6-PACK programme, replicated what was developed and evaluated in the single centre observational study. ${ }^{25}$ Appendix 1 gives a detailed description of the 6-PACK programme according to the CONSORT extension template for intervention description and replication guidelines. In brief, the programme included a nine item fall risk tool, ${ }^{20}$ as well as six interventions: "falls alert" sign, supervision of patients in the bathroom, ensuring patients' walking aids are within reach, establishment of a toileting regimen, use of a low-low bed, and use of a bed/chair alarm. Nurses were asked to update the fall risk tool for each of their patients each shift and to apply a falls alert sign and one or more of the remaining 6-PACK interventions to patients classified as being at high risk. Selection of interventions for each patient was 
based on clinical judgment. The 12 intervention wards implemented the 6-PACK programme over a 12 month period. Implementation was supported by project change management and programme facilitators, a standardised implementation guide, a hospital based site clinical leader, and ward champions who conducted staff education and audits and provided reminders and feedback on the use of programme components during the trial period.

\section{Comparator}

Control wards were asked to continue with usual care throughout the trial period. Usual care involved falls prevention practices provided by wards as part of existing hospital policy, which may have included some components of the 6-PACK programme and other interventions such as non-slip socks, constant patient observers, and falls alert wrist bands.

\section{Outcome measures}

The co-primary outcomes were falls and fall injuries per 1000 occupied bed days. We defined a fall as "an event resulting in a person coming to rest inadvertently on the ground, floor, or other lower level."28 We defined a fall injury as any reported physical harm resulting from a fall (including bruising, abrasions, lacerations, and fractures) with the possibility of multiple injuries per fall. Data on injurious falls (a fall resulting in one or more injuries) and fractures (vertebral and peripheral) were also reported. Fractures were verified by medical imaging.

\section{Data collection}

Falls, falls prevention practices, and patients' demographic data were prospectively recorded for a three month period before the trial to inform implementation of the 6-PACK programme and provide a baseline reference period with which the sample's characteristics and outcomes for the 12 month study period could be compared. Admitted patients' demographic characteristics and diagnoses came from hospital administrative datasets. Diagnoses were recorded using the ICD-10-AM (international classification of diseases, 10th revision, Australian modification). We applied published algorithms to generate Charlson comorbidities from ICD10-AM diagnoses. ${ }^{29}$ Falls data were prospectively collected via daily audit of patients' medical records and verbal reports from the ward nurse unit manager about falls known to have occurred within the previous 24 hours, as well as monthly audit of hospital incident reporting and administrative databases. We reviewed radiological investigation reports to verify fractures. A second independent assessor reviewed and re-coded all recorded falls (ward, injuries sustained, fall location, time, and activity), and disagreements were resolved by a third. We included as an outcome event any fall recorded in any source that was verified (met the study definition and occurred on a study ward during the study period) by the second independent assessor. Process data on completion of the risk tool and use of the 6-PACK programme components were recorded by daily medical record audit and structured bedside observation by trained assessors using a standardised tool during the baseline and study period.

\section{Sample size}

The sample size estimation was conservatively based on an unpaired analysis approach despite a paired analysis being the a priori planned analysis. ${ }^{27}$ This was based on the knowledge that the ward matching may not be successful owing to the experience of a similar previous trial. ${ }^{30}$ The sample size estimate assumed a fall injury rate in control wards of 2.8 per 1000 occupied bed days, an average of 8500 occupied bed days per ward in 12 months, and an average cluster (ward) size of 1000 patient ward admissions. The required number of fall injuries in the control group, $\mathrm{D}_{0}$, was calculated from a formula that assumes a Poisson distribution: $\mathrm{D}_{0}=\mathrm{Z}^{2}(\theta+1) / \theta\left(\log _{\mathrm{e}} \theta\right)^{2}$, where the rate ratio $\theta=0.7, z=2.8$, and an inflation factor of 2 was applied for the cluster design effect on the basis of an intraclass correlation coefficient of $0.001 .^{30}$ For $80 \%$ power to detect a rate ratio of 0.7 between intervention and control wards at the $5 \%$ significance level, 12 intervention and 12 control wards were needed, assuming a total of 510 fall injuries, from approximately 212000 occupied bed days during the trial period. ${ }^{27}$

\section{Randomisation}

After recruitment of wards and collection of the three month baseline data, ward pairs were defined either by being the two wards of the same type recruited at a hospital or, in the case of two hospitals where four wards of the same type were recruited, by having the most comparable baseline fall injury rates. After this, a statistician blinded to the names of the hospitals and wards randomised one ward from each pair to receive the intervention or to continue with usual care (control). In fact, in only three of the 12 pairs were baseline fall injury rates actually similar. We used the RALLOC command in Stata to develop the randomisation schedule, using a random sequence in blocks of two generated by the study statistician. ${ }^{31}$ Concealment of allocation was ensured, as the schedule was accessible only by the study statistician, who was not involved in ward recruitment or data collection.

\section{Blinding}

As the intervention was nurse led, blinding of ward nurses and patients was not possible. Blinding of the assessors collecting the fall and falls prevention practice data was also not possible. Assessors blinded to group allocation did the secondary coding of characteristics of falls and injuries, and the primary assessor completed the coding. A statistician blinded to group allocation (RW) did the data analysis.

\section{Statistical methods}

We linked hospital administrative datasets that contained demographic and diagnosis data to fall data (linking variables were patient's identifier, date of hospital admission, and ward name). We used descriptive statistics to profile the sample; fall, fall injury, and injurious fall rates; and process data on the use of 6-PACK programme components (sum of total components used each observed day; 
maximum=7). To evaluate the efficacy and implementation of the programme, we compared rates of falls, fall injuries, and use of 6-PACK components between the intervention and control groups by using mixed effect negative binomial models, with the at risk time being each ward admission's length of stay. Primary analyses allowed for pairing of wards by inclusion of a fixed effect for ward type (surgical or medical) and clustering of a ward's admissions by inclusion of a random effect for ward. We did not include a fixed effect for ward pairing as recommended by Martin et al, ${ }^{32}$ as fall injury rates for the baseline period were not particularly similar within ward pairs (matching correlation was 0.14).

We did a sensitivity analysis with ward pair as a fixed effect corresponding to the a priori analysis plan. Secondary analyses included adjustment in the same model framework for the pre-specified variables of age and cognitive impairment. ${ }^{27}$ We did subgroup analysis by ward type by including an interaction term between ward type and randomised group in each of the fall and fall injury models. We investigated potential contamination by examining changes in the use of 6-PACK programme components from the baseline to the randomised controlled trial period in the control wards. Post hoc analysis explored the timing of the use of 6-PACK programme components with a 100\% adherence target (risk assessment completed for all patients, falls alert sign and at least one other 6-PACK intervention in place for high risk patients) and falls on intervention wards during the trial period. We calculated the percentage adherence for programme components and the percentage of falls on each of the first five days of the ward stay. We used a significance level of $\mathrm{P}<0.05$ to indicate statistical significance, and all analyses applied intention to treat principles. We used Stata MP 13 statistical software for all analyses.

\section{Patient involvement}

No patients were involved in setting the research question or the outcome measures, nor were they involved in recruitment, or the design and implementation of the study. There are no plans to involve patients in dissemination.

\section{Results}

No major protocol deviations or unexpected adverse events occurred during the study period.

\section{Characteristics of wards and participants}

This trial involved 46245 ward admissions, from 16 medical wards and eight surgical wards, during the period January 2012 to March 2013. Characteristics of admitted patients and length of stay were similar for intervention and control groups and across baseline and randomised controlled trial periods (table 1). In all, 31411 unique patients were admitted to the wards, including $3853(12.3 \%)$ patients admitted to both an intervention and a control ward at different times during the trial period. Eighteen of the 24 wards had a trial period of 12 months (fig 1 ). Three ward pairs had an 11 month trial period owing to ward closures.

The use of all 6-PACK programme components (fall risk tool and six interventions) was threefold higher on intervention wards than on control wards (incidence rate ratio 3.05, 95\% confidence interval 2.14 to $4.34 ; \mathrm{P}<0.001$ ). Use of 6-PACK programme components increased rapidly on the intervention wards after introduction of the programme at the start of the randomised trial period. No change was observed on the control wards, suggesting that contamination was unlikely. The higher use of 6-PACK programme components was sustained for the eight months of the trial period for which implementation data were collected (fig 2). Budget constraints prevented collection of these data for the last four months of the trial period. Use of individual components of the 6-PACK programme was higher on the intervention wards than on the control wards during the trial period (fig 3).

During the baseline period, 393 falls and 144 fall injuries were recorded for 273 fallers contributing 284 admissions. The rates of falls and fall injuries were 6.45 (95\% confidence interval 5.84 to 7.11 ) and 2.36 (2.01 to 2.78) per 1000 occupied bed days.

During the randomised trial period, 1831 falls and 613 fall injuries were recorded for 1247 fallers contributing 1333 admissions (table 2). The fall rate in intervention wards was 7.46 (7.00 to 7.50) per 1000 occupied bed days compared with 7.03 (6.59 to 7.51) per 1000 occupied bed days in control wards, and the rate of fall injuries was 2.33 (2.07 to 2.83) and 2.53 (2.26 to 2.82) per 1000 occupied bed days, respectively. The fall and fall injury rates varied across wards and ward types (appendix 2). Rates of falls, fall injuries, and injurious falls remained stable across the baseline and trial period for both groups (fig 4).

The rates of falls (incidence rate ratio 1.04, 0.78 to 1.37; $\mathrm{P}=0.796)$ and fall injuries (0.96, 0.72 to 1.27 ; $\mathrm{P}=0.766$ ) were similar in intervention and control wards during the randomised trial period. Consistent results were observed in analyses by ward type (medical or surgical), both in primary unadjusted analyses and secondary analyses adjusted for age and cognitive impairment (appendix 3). In the sensitivity analysis that included ward pair as a fixed effect, the rates of falls (incidence rate ratio 1.04, 0.92 to 1.18; $\mathrm{P}=0.542$ ) and fall injuries (0.96, 0.77 to 1.20 ; $\mathrm{P}=0.734$ ) were similar in intervention and control wards during the randomised trial period. Visual inspection of temporal trends in fall (appendix 4) and fall injury rates (appendix 5) by ward also found no discernible trends in individual wards throughout the 12 month trial period.

Figure 5 outlines the timing of use of 6-PACK programme components for which there was a 100\% adherence target and falls for the first five days of ward admission for intervention wards only. Completion of the risk tool was relatively stable across the first five days of ward admission, whereas use of alert signs and other interventions tended to increase. Falls most commonly occurred on the second day of ward admission.

\section{Discussion}

The introduction of the 6-PACK programme improved completion of a fall risk tool and use of fall prevention interventions recommended by best practice guidelines but had no effect on falls or fall injuries compared with usual care. Falls remain a frequent and substantial 
Table 1 | Characteristics of intervention and control wards and patients. Values are numbers (percentages) unless stated otherwise

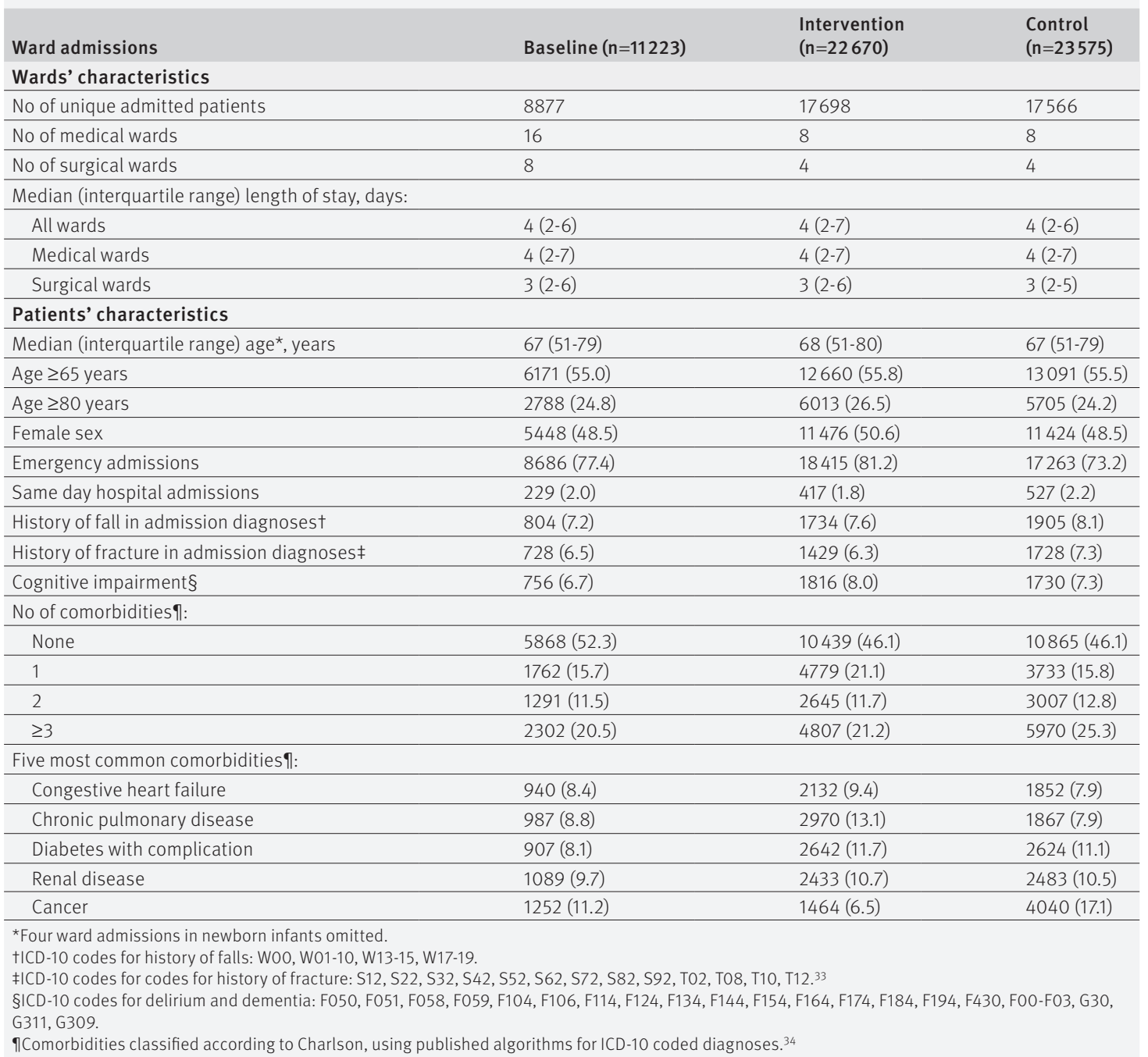

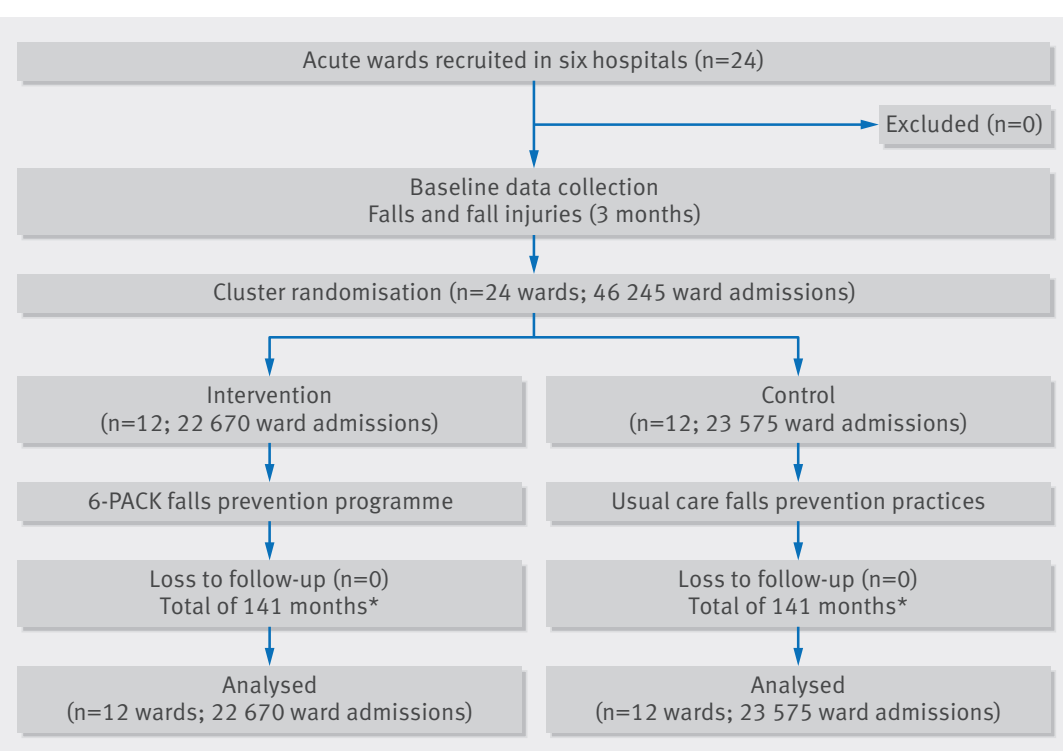

Fig 1 | Flow of wards and patients through trial. *Three ward pairs ceased observation at 11 months owing to ward closures source of harm for older people admitted to acute hospitals. Rates for some wards in this trial approached 18 per 1000 occupied bed days for falls and 4 per 1000 occupied bed days for fall injuries, and overall rates are comparable to rates reported in other multicentre trials. ${ }^{2630}$

This study tested a falls prevention programme that was developed, implemented, and refined as part of usual care, with use of quality improvement methods, and for which evidence of positive effects on fall injuries existed from a single centre observational evaluation. ${ }^{25}$ Although many aspects of the 6-PACK programme represent practices that are used in many acute wards, recent reports highlight that current falls prevention practice in acute hospitals is suboptimal. ${ }^{124}$ The aim of the 6-PACK programme was to increase the frequency with which the 6-PACK interventions were used on the premise that increased use would decrease falls and associated harm. The trial was successful at increasing the use of these interventions, and we also found evidence of the timeliness of delivery, with a high proportion of patients receiving interventions within the first day of their ward admission. This suggests that the 6-PACK implementation strategy was effective at 


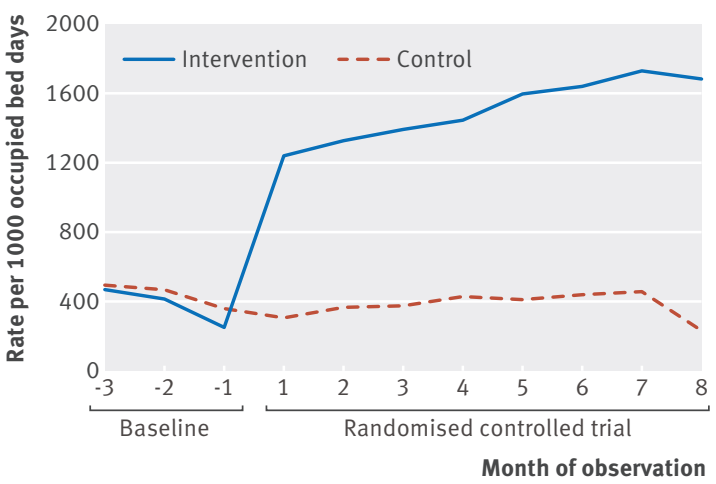

Fig 2 | Rate of use of 6-PACK programme components (fall risk tool and six interventions - that is, maximum uptake of 7 per occupied bed day) by month and group

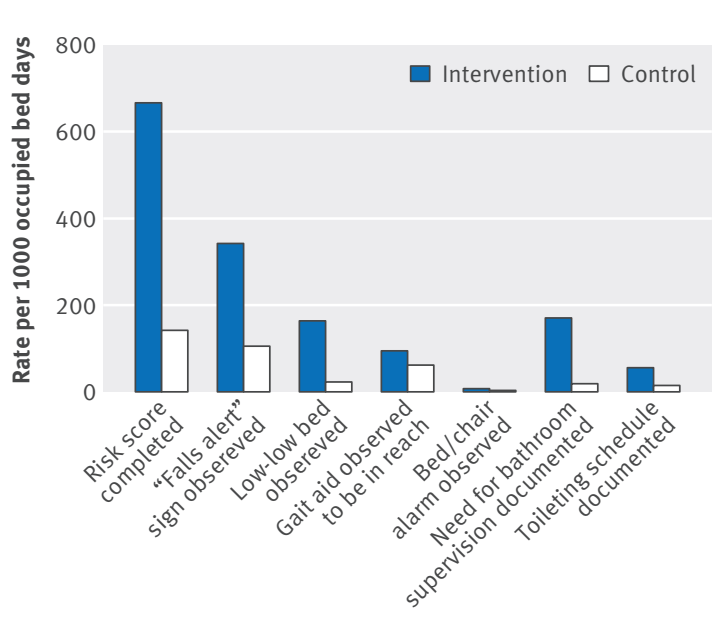

Fig 3 | Use of 6-PACK programme components during randomised controlled trial period by group

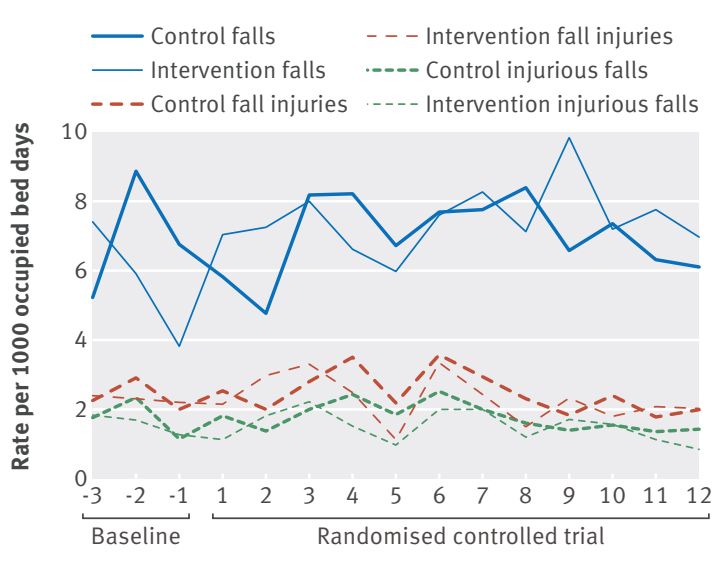

Month of observation

Fig 4 | Fall outcomes by month and group

achieving meaningful changes in falls prevention practice and that the risk of implementation failure was low. However, data are lacking on the use of falls prevention interventions in previous studies to which this study can be benchmarked so that the level of effectiveness of the implementation can be more fully appreciated. Furthermore, the selection of interventions (except for the falls alert sign) was based on the clinical judgment of the patients' treating nurse(s). Therefore, we did not

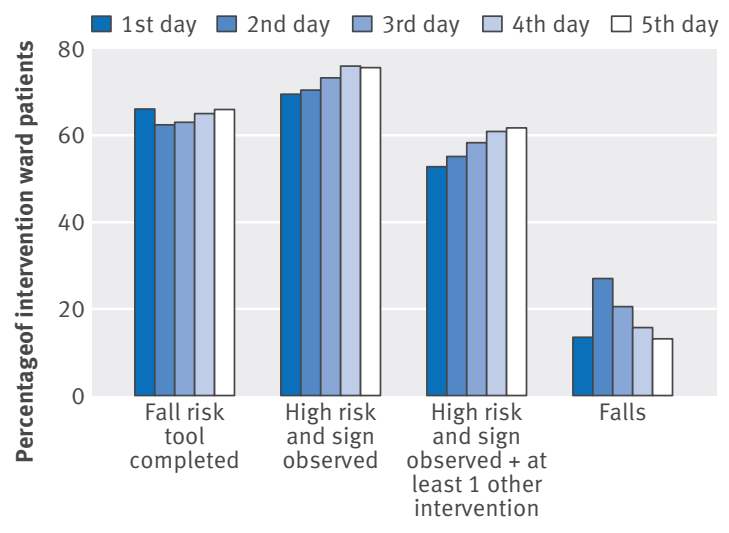

Fig 5 | Use of 6-PACK programme components with $100 \%$ adherence target and falls by day of ward admission for intervention ward patients

expect that $100 \%$ of patients at high risk would receive the remaining five interventions. Appropriate use of interventions depended on case mix. Although further exploration of the implementation fidelity-including adherence to the programme and its moderating factors (hospital resources, implementation strategies, and staff responsiveness)-is planned, this analysis suggests that implementation was effective.

Possible explanations for our finding of no effect include contamination, confounding, and ineffectiveness of the intervention. The results presented provide evidence that the risk of contamination was low, as the use of 6-PACK programme components was significantly lower on control wards and rates were consistent with those recorded in the pre-trial baseline period. The groups were comparable for key measured factors known to affect risk of falls, including age and cognitive impairment, suggesting that confounding due to variation in case mix was also unlikely, although there may be confounding due to imbalance between randomised groups in factors not measured (including staff skill mix; availability of mobility aids; use of benzodiazepines; falls risk factors including gait, balance, muscle strength, functional impairment, and use of walking aids; and complexity of illness).

In the absence of evidence of implementation failure, contamination, or confounding, we conclude that the 6-PACK programme was not effective at preventing falls or fall injuries. Reasons for the lack of effect may relate to the interventions included in the 6-PACK programme. Recent studies suggest that several of the individual 6-PACK programme components may be ineffective. An observational study indicated that low-low beds may reduce fall injuries, ${ }^{23}$ but a larger randomised controlled trial reported no effect. ${ }^{35}$ Recent trials have also cast doubt on the effectiveness of bed/chair alarms. ${ }^{3637}$ Falls prevention programmes may need a greater focus on preventing delirium, which was not specifically covered in the 6-PACK programme. Previous studies report that one in two falls occur in patients who are confused. ${ }^{2238}$ The successful FallSafe programme included urinalysis screening and reducing night time sedation to tackle delirium. ${ }^{26}$

Although the interventions included in 6-PACK hold face validity, they may lack potency to effectively mitigate 


\begin{tabular}{|c|c|c|c|}
\hline Ward admissions & $\begin{array}{l}\text { Baseline } \\
(\mathrm{n}=11223)\end{array}$ & $\begin{array}{l}\text { Intervention } \\
(\mathrm{n}=22670)\end{array}$ & $\begin{array}{l}\text { Control } \\
(n=23575)\end{array}$ \\
\hline Unique admitted patients & 8877 & 17698 & 17566 \\
\hline Falls & 393 & 937 & 894 \\
\hline Fallers & $284(3.2)$ & $655(3.7)$ & $678(3.9)$ \\
\hline Admissions with $\geq 2$ falls & $53(0.5)$ & $144(0.6)$ & $131(0.6)$ \\
\hline Injurious falls* & $104(26.5)$ & $196(20.9)$ & $229(25.6)$ \\
\hline Fall injuriest: & 144 & 292 & 321 \\
\hline Skin injury, open & 79 (54.9) & $177(60.6)$ & $186(57.9)$ \\
\hline Skin injury, closed§ & $50(34.7)$ & $90(30.8)$ & $113(35.2)$ \\
\hline Sprain & $3(2.1)$ & $1(0.3)$ & $0(0)$ \\
\hline Dislocation & $1(0.7)$ & $0(0)$ & $0(0)$ \\
\hline Fractures (all): & $9(6.3)$ & $12(4.1)$ & $14(4.4)$ \\
\hline Vertebral fractures & $1(0.7)$ & $1(0.3)$ & $1(0.3)$ \\
\hline Non-vertebral fractures & $8(5.6)$ & $11(3.8)$ & $13(4.0)$ \\
\hline Subdural haematoma & $0(0)$ & $4(1.4)$ & $3(0.9)$ \\
\hline $\begin{array}{l}\text { *Count of all falls resulting in inj } \\
\text { †Count of all injuries resulting fr } \\
\neq \text { Graze, laceration, skin tear. } \\
\text { §Bruise, haematoma. }\end{array}$ & & & \\
\hline
\end{tabular}

the risk of falls and injury. The findings of a recent meta-analysis support the concept of a lack of potency of multifactorial falls prevention approaches-assuming a rate of 8 falls per 1000 occupied bed days, the number needed to treat to prevent a single inpatient fall is 1250 patient days. ${ }^{39}$ This represents one fall prevented every six weeks in a standard 30 bed ward and assumes a small treatment effect of 0.9 (odds ratio) generated from pooled effects of five non-randomised clinical trials and one randomised trial. Potency may be limited by a reliance on behavioural change from both the nurses and the patients.

\section{Strengths and limitations of study}

This study, involving 24 wards from six hospitals, is the largest randomised controlled trial of falls prevention to have been undertaken to date and, importantly, was powered to detect changes in fall injuries. We used a rigorous, cluster randomised design with concealment of allocation, inclusion of all patients admitted to study wards, collection of falls data from multiple sources, collection of data on implementation, blinded outcome assessment, and intention to treat analysis. ${ }^{1242630}$

Study limitations warrant consideration. Importantly, hospital sampling was done at convenience. The participating hospitals may therefore not be representative of the broader population of acute hospitals in Australia or internationally. The assessment of cognitive impairment was based on ICD-10 codes recorded in routine hospital datasets, which are likely to have been under-coded. ${ }^{40} \mathrm{We}$ used multiple data sources to identify primary outcomes, but falls and fall injuries may have been reported more on intervention wards owing to increased awareness of falls with 6-PACK implementation. However, we saw no significant change in reporting of falls outcomes between the baseline and intervention periods on the control or intervention wards. Similarly, although process data on the use of 6-PACK interventions were recorded by assessors who were aware of group allocation, rates of use of 6-PACK component in control wards were comparable across the baseline period before wards were randomised, and group allocation was revealed, and the randomised trial period when ward allocation was known. Therefore, we consider that the risk of reporting or detection bias in this study was low. All fall events were also double coded (event meets fall definition, injuries sustained, ward and fall characteristics) by a second assessor who was blind to the ward allocation and the codes assigned by the first assessor to minimise reporting bias.

\section{Comparison with other studies}

The findings of this study are in accordance with those of previous randomised controlled trials and observational studies, which together provide increasing evidence of no effect for falls prevention interventions in the acute hospital setting. The Cochrane review published in 2012 included four multiple and eight single intervention studies in acute hospital samples and concluded that limited evidence existed to support any one intervention. ${ }^{14}$ Of the four multiple intervention studies, two showed no effect on falls, ${ }^{3041}$ and two reported a reduction in falls. ${ }^{1642}$ Both trials that reported a reduction in falls were single centre in design, recruited individual patients to participate in the study (rather than including all patients admitted to study wards), required additional staffing to implement the intervention, and collected falls data from a single source only. These factors limit the generalisability and validity of the findings of both of these studies to the broader acute care population. The method for collecting falls data for hospital based trials should incorporate multiple sources. ${ }^{43}$ Recruitment of individual patients to a study often results in a sample with under-representation of patients who are acutely unwell, require interpreters, or have cognitive impairment, therefore limiting generalisability. In addition, the study by Stenvall et al included only patients with hip fractures and reported a total postoperative observation period of 2860 days for the 102 intervention participants, ${ }^{16}$ suggesting that the study population was more representative of the subacute setting. Further trials are needed to confirm the effects reported in these two studies.

The eight single intervention studies in acute populations included in the Cochrane review also found no effect on falls. They tested bed exit alarms, ${ }^{44}$ a behavioural advisory service for people with cognitive impairment, ${ }^{45}$ multifaceted guideline implementation, ${ }^{4647}$ an acute care for the elderly service, ${ }^{48}$ a computer based falls prevention tool kit, ${ }^{49}$ multimedia patient education, ${ }^{50}$ and low-low beds. ${ }^{35}$ Two additional randomised controlled trials have investigated single interventions for falls prevention in acute wards and were not included in the Cochrane review. One tested bed exit alarms and found no effect on falls ${ }^{36}$; the other tested shock absorbing flooring and reported fewer falls with injury. ${ }^{51}$ Although these latter results highlight the potential benefit of shock absorbing flooring, the study was only a pilot trial including a small number of patients, so a larger trial is needed to confirm the effects.

A recent review that included eight randomised and non-randomised comparative studies in US acute hospitals also reported that multifactorial interventions had 
no effect on falls and concluded that further research is needed to provide evidence on how hospitals can successfully prevent falls. ${ }^{52}$ This finding is consistent with a review of 17 international randomised and non-randomised comparative studies, of which 13 included acute wards, which concluded that there was a lack of evidence for reducing falls in hospital populations with a shorter length of stay. ${ }^{18} \mathrm{~A}$ frequent limitation of previous studies is that they have been underpowered to detect differences in rates of fall injury.

\section{Conclusions and policy implications}

A pressing need exists to identify novel methods to reduce harm from falls in acute hospitals. Given that falls occur in seconds and have been difficult to prevent, other innovative approaches, such as ward redesign or intelligent sensor systems (to improve observation of high falls risk patients), 53 programmes that target the behavioural and psychological symptoms of dementia and delirium, rationalising drugs, post-fall huddles, programmes to increase patient engagement, or a whole system approach to patient safety and care of older vulnerable people who are acutely unwell, may offer new solutions and warrant further investigation. The updated 2013 clinical guideline on falls from the National Institute for Health and Care Excellence also supports the concept of exploring environmental adaptations aimed at reducing the risk of falling in older inpatients. ${ }^{5}$

The findings of this study and those of other similar trials that have reported findings of no effect raise questions about the expenditure of staff time on the delivery of interventions that have been shown to be ineffective. ${ }^{3036}$ The increase in completion of the fall risk tool and delivery of fall prevention interventions observed in this study would seem to be a positive outcome, but these activities may take time away from other fall prevention or care activities. The "opportunity costs" of delivering falls prevention interventions known to be ineffective are considerable, and disinvestment in these practices should be considered. Interventions such as providing assistance in the bathroom to those who need it, toileting schedules, and ensuring a gait aid is in reach represent basic care for patients and should not be discontinued. However, evidence is lacking to show that these activities prevent falls or associated harm. No evidence exists to support use of low-low beds, bed/chair alarms, falls alert signs, or fall risk tools, so the use of these interventions must be questioned in an environment that is time and resource poor with many competing priorities. The findings of this trial support recent recommendations relating to the US non-payment for serious in-hospital fall injuries that policy makers should implement only outcome metrics that have robust evidence on how to improve them. ${ }^{13}$

In conclusion, greater use of the components within the 6-PACK falls prevention programme had no effect on falls or fall injuries in the 12 acute intervention wards that participated in this trial. Although the substantial harm and negative consequences of inpatient falls are unquestionable, high quality evidence showing the effectiveness of preventive interventions for falls in acute wards is lacking. Epidemiological data are also lacking to support the idea that the increased focus on falls prevention in hospitals via introduction of guidelines, accreditation standards, and quality improvement initiatives has had any effect on falls or their consequences. System level interventions, environmental interventions, or both may offer improved potency and effectiveness and should now be the focus of further investigation.

We acknowledge the contribution of Sheral Rifat and Darshini Ayton in the preparation of this article; the Injury Prevention Unit, The Northern Hospital, Northern Health, Melbourne, Australia, who developed the 6-PACK programme; and the late Damien Jolley, who provided substantial input to the design of the 6-PACK project. The study could not have been completed without the collaboration and support from the participating hospitals, site clinical leaders, and nursing staff. Contributors: ALB led the conception, design, and application for funding of the project in conjunction with $C A B, T P H, K D H, S G B, M B$, RGC, PML, CS, and SZ. ALB, RTM, JK, CAB, TPH, KDH, SGB, MB, RGC, $P M L, C S$, and $S Z$ participated in the steering committee and project governance. RIL was a study site investigator. ALB led the drafting all sections of this manuscript except the results in consultation with all of the co-authors. RTM managed the collection and verification of data and led the drafting of the results section of this manuscript. RW was responsible for the analysis and interpretation of data. All authors reviewed and revised the manuscript critically for important intellectual content. ALB is the guarantor.

Funding: This project was funded by the National Health and Medical Research Council (NHMRC), Australia (APP1007627). ALB's salary was funded by a career development fellowship from the NHMRC (APP1067236). RTM's salary was supported by a postgraduate scholarship from the NHMRC (APP1055604). TPH's salary was funded by a career development fellowship from the NHMRC (APP1069758). CS's salary was funded by a career development fellowship from the NHMRC (APP632929). The funders of the study had no role in study design, data collection, data analysis, data interpretation, or writing of the manuscript. ALB, RTM, and RW had full access to all the data in the study, and the corresponding author had final responsibility for the decision to submit for publication.

Competing interests: All authors have completed the ICMJE uniform disclosure form at www.icmje.org/coi_disclosure.pdf and declare: no support from any organisation for the submitted work other than that detailed above; no financial relationships with any organisations that might have an interest in the submitted work in the previous three years; no other relationships or activities that could appear to have influenced the submitted work.

Ethical approval: This study was approved by Monash University Human Research Ethics Committee (CF11/0229, 2011000072) and each of the relevant hospital ethics committees.

Data sharing: A full dataset that includes patient level data, technical appendix, and statistical code is available on reasonable request from the corresponding author. Individual patient consent was not obtained but the presented data are anonymised and risk of identification is low. Transparency statement: The lead author (the manuscript's guarantor) affirms that the manuscript is an honest, accurate, and transparent account of the study being reported; that no important aspects of the study have been omitted; and that any discrepancies from the study as planned (and, if relevant, registered) have been explained.

This is an Open Access article distributed in accordance with the Creative Commons Attribution Non Commercial (CC BY-NC 3.0) license, which permits others to distribute, remix, adapt, build upon this work non-commercially, and license their derivative works on different terms, provided the original work is properly cited and the use is noncommercial. See: http://creativecommons.org/licenses/by-nc/3.0/.

Royal College of Physicians. Report of the 2011 inpatient falls pilot audit.RCP, 2012

2 Jørgensen TS, Hansen AH, Sahlberg M et al. Nationwide time trends and risk factors for in-hospital falls-related major injuries. Int J Clin Pract 2015;69: 703-9. doi:10.1111/ijcp.12624

3 Wong CA, Recktenwald AJ, Jones ML, Waterman BM, Bollini ML, Dunagan WC. The cost of serious fall-related injuries at three Midwestern hospitals. Jt Comm / Qual Patient Saf 2011;37: 81-7.

4 Morello RT, Barker AL, Watts IJ et al. The extra resource burden of in-hospital falls: a cost of falls study. Med J Aust 2015;203: 367-76. doi:10.5694/mja15.00296

5 National Institute for Health and Care Excellence. Falls: assessment and prevention of falls in older people. NICE, 2013. 
6 Australian Commission on Safety and Quality in Health Care. Preventing falls and harm from falls in older people: best practice guidelines for Australian hospitals. ACSQHC, 2009.

7 American Geriatrics Society, British Geriatrics Society. AGS/BGS clinical practice guideline: prevention of falls in older persons.American Geriatrics Society, 2010.

8 Center for Performance Sciences. Acute care implementation manual: international quality indicator project. Centre for Performance Sciences, 2006

9 Australian Council on Healthcare Standards. National Safety and Quality Health Service Standards Program. ACHS, 2012.

10 Power M, Stewart K, Brotherton A. What is the NHS safety thermometer? Clin Risk 2012;18: 163-9. doi:10.1258/cr.2012.012038

11 Rosenthal MB. Nonpayment for performance? Medicare's new reimbursement rule. N Engl J Med 2007;357: 1573-5. doi:10.1056/ NEJMp078184

12 Waters TM, Daniels MJ, Bazzoli GJ et al. Effect of Medicare's nonpayment for Hospital-Acquired Conditions: lessons for future policy. JAMA Intern Med 2015;175: 347-54. doi:10.1001/ jamainternmed 2014.5486

13 Umscheid CA, Brennan PJ. Incentivizing “structures" over “outcomes" to bridge the knowing-doing gap. JAMA Intern Med 2015;175: 354-5. doi:10.1001/jamainternmed.2014.5293

14 Cameron ID, Gillespie LD, Robertson MC et al. Interventions for preventing falls in older people in care facilities and hospitals. Cochrane Database Syst Rev 2012;12: CD005465

15 Haines TP, Bennell KL, Osborne RH, Hill KD. Effectiveness of targeted falls prevention programme in subacute hospital setting: randomised controlled trial. BMJ 2004;328: 676. doi:10.1136/bmj.328.7441.676

16 Stenvall M, Olofsson B, Lundström M et al. A multidisciplinary, multifactorial intervention program reduces postoperative falls and injuries after femoral neck fracture. Osteoporos Int 2007;18: 167-75. doi:10.1007/s00198-006-0226-7

17 Hill AM, McPhail SM, Waldron $\mathrm{N}$ et al. Fall rates in hospital rehabilitation units after individualised patient and staff education programmes: a pragmatic, stepped-wedge, cluster-randomised controlled trial. Lancet 2015;385: 2592-9. doi:10.1016/ S0140-6736(14)61945-0

18 Oliver D, Healey F, Haines TP. Preventing falls and fall-related injuries in hospitals. Clin Geriatr Med 2010;26: 645-92. doi:10.1016/j. cger.2010.06.005

19 Australian Institute of Health and Welfare. Australian hospital statistics 2011-12.AlHW, 2013

20 Barker A, Kamar J, Graco M, Lawlor V, Hill K. Adding value to the STRATIFY falls risk assessment in acute hospitals. J Adv Nurs 2011;67 450-7. doi:10.1111/j.1365-2648.2010.05503.x

21 Evans D, Hodgkinson B, Lambert L, Wood J. Falls risk factors in the hospital setting: a systematic review. Int / Nurs Pract 2001;7: 38-45. doi:10.1046/j.1440-172x.2001.00269.x

22 Hitcho EB, Krauss MJ, Birge $S$ et al. Characteristics and circumstances of falls in a hospital setting: a prospective analysis. J Gen Intern Med 2004:19: 732-9. doi:10.1111/j.1525-1497.2004.30387x

23 Barker A, Kamar J, Tyndall T, Hill K. Reducing serious fall-related injuries in acute hospitals: are low-low beds a critical success factor?] Adv Nurs 2013:69:112-21. doi:10.1111/j.1365-2648.2012.05997x

24 Barker A. An evaluation of the preventing falls and harm from falls in older people best practice guidelines for Australian hospitals. Monash University, 2012

25 Barker A, Kamar J, Morton A, Berlowitz D. Bridging the gap between research and practice: review of a targeted hospital inpatient fal prevention programme. Qual Saf Health Care 2009;18: 467-72. doi:10.1136/qshc.2007.025676

26 Healey F, Lowe D, Darowski A et al. Falls prevention in hospitals and mental health units: an extended evaluation of the FallSafe quality improvement project. Age Ageing 2014;43: 484-91. doi:10.1093/ ageing/aft190

27 Barker A, Brand C, Haines T et al. The 6-PACK programme to decrease fall-related injuries in acute hospitals: protocol for a cluster randomised controlled trial. Inj Prev 2011;17: e5. doi:10.1136 injuryprev-2011-040074

28 Hauer K, Lamb SE, Jorstad EC, Todd C, Becker C. PROFANE-Group. Systematic review of definitions and methods of measuring falls in randomised controlled fall prevention trials. Age Ageing 2006;35: 5-10. doi:10.1093/ageing/afiz218

29 Sundararajan V, Henderson T, Perry C, Muggivan A, Quan H, Ghali WA New ICD-10 version of the Charlson comorbidity index predicted in-hospital mortality. J Clin Epidemiol 2004;57: 1288-94. doi:10.1016/j.jclinepi.2004.03.012

30 Cumming RG, Sherrington C, Lord SR et al. Prevention of Olde People's Injury Falls Prevention in Hospitals Research Group. Cluster randomised trial of a targeted multifactorial intervention to prevent falls among older people in hospital. BMJ 2008;336: 758-60. doi:10.1136/bmj.39499.546030.BE
31 Ryan P. Ralloc: Stata module to design randomized controlled trials.Statistical Software Components, 2011.

32 Martin DC, Diehr P, Perrin EB, Koepsell TD. The effect of matching on the power of randomized community intervention studies. Stat Med 1993;12: 329-38. doi:10.1002/sim.4780120315

33 Brand CA, Sundararajan V. A 10-year cohort study of the burden and risk of in-hospital falls and fractures using routinely collected hospital data. Qual Saf Health Care 2010;19: e51.

34 Quan H, Sundararajan V, Halfon P et al. Coding algorithms for defining comorbidities in ICD-9-CM and ICD-10 administrative data. Med Care 2005;43: 1130-9. doi:10.1097/01.mlr.0000182534.19832.83

35 Haines TP, Bell RA, Varghese PN. Pragmatic, cluster randomized trial of a policy to introduce low-low beds to hospital wards for the prevention of falls and fall injuries. J Am Geriatr Soc 2010;58: 435-41. doi:10.1111/j.1532-5415.2010.02735.x

36 Shorr RI, Chandler AM, Mion LC et al. Effects of an intervention to increase bed alarm use to prevent falls in hospitalized patients: cluster randomized trial. Ann Intern Med 2012;157: 692-9. doi:10.7326/0003-4819-157-10-201211200-00005

37 Sahota O, Drummond A, Kendrick D et al. REFINE (REducing Falls in In-patieNt Elderly) using bed and bedside chair pressure sensors linked to radio-pagers in acute hospital care: a randomised controlled trial. Age Ageing 2014;43: 247-53. doi:10.1093/ageing/ aft155

38 Schwendimann R, Bühler H, De Geest S, Milisen K. Characteristics of hospital inpatient falls across clinical departments. Gerontology 2008:54: 342-8. doi:10.1159/000129954

39 DiBardino D, Cohen ER, Didwania A. Meta-analysis: multidisciplinary fall prevention strategies in the acute care inpatient population. J Hosp Med 2012;7: 497-503. doi:10.1002/jhm.1917

40 Cummings E, Maher R, Showell CM et al. Hospital coding of dementia: is it accurate?HIM / 2011;40: 5-11.

41 Healey F, Monro A, Cockram A, Adams V, Heseltine D. Using targeted risk factor reduction to prevent falls in older in-patients: a randomised controlled trial. Age Ageing 2004;33: 390-5. doi:10.1093/ageing/ afh130

42 Ang E, Mordiffi SZ, Wong HB. Evaluating the use of a targeted multiple intervention strategy in reducing patient falls in an acute care hospital: a randomized controlled trial. J Adv Nurs 2011;67: 1984-92. doi:10.1111/j.1365-2648.2011.05646.x

43 Hill AM, Hoffmann T, Hill K et al. Measuring falls events in acute hospitals-a comparison of three reporting methods to identify missing data in the hospital reporting system. J Am Geriatr SoC 2010;58: 1347-52. doi:10.1111/j.1532-5415.2010.02856.x

44 Tideiksaar R, Feiner CF, Maby J. Falls prevention: the efficacy of a bed alarm system in an acute-care setting. Mt Sinai J Med 1993;60: 522-7.

45 Mador JE, Giles L, Whitehead C, Crotty M. A randomized controlled trial of a behavior advisory service for hospitalized older patients with confusion. Int J Geriatr Psychiatry 2004;19: 858-63. doi:10.1002/ gps.1165

46 Koh SL, Hafizah N, Lee JY, Loo YL, Muthu R. Impact of a fall prevention programme in acute hospital settings in Singapore. Singapore Med J 2009; 50: 425-32

47 van Gaal BG, Schoonhoven L, Mintjes JA et al. Fewer adverse events as a result of the SAFE or SORRY? programme in hospitals and nursing homes. part i: primary outcome of a cluster randomised trial. Int J Nurs Stud 2011;48: 1040-8. doi:10.1016/j.ijnurstu.2011.02.017

48 Wald HL, Glasheen IJ, Guerrasio J, Youngwerth IM, Cumbler EU. Evaluation of a hospitalist-run acute care for the elderly service. $J$ Hosp Med 2011;6: 313-21. doi:10.1002/jhm.906

49 Dykes PC, Carroll DL, Hurley A et al. Fall prevention in acute care hospitals: a randomized trial. JAMA 2010;304: 1912-8. doi:10.1001/ jama.2010.1567

50 Haines TP, Hill AM, Hill KD et al. Patient education to prevent falls among older hospital inpatients: a randomized controlled trial. Arch Intern Med 2011:171: 516-24. doi:10.1001/archinternmed.2010.444

51 Drahota AK, Ward D, Udell JE et al. Pilot cluster randomised controlled trial of flooring to reduce injuries from falls in wards for older people. Age Ageing 2013;42: 633-40. doi:10.1093/ageing/aft067

52 Hempel S, Newberry S, Wang Z et al. Hospital fall prevention: a systematic review of implementation, components, adherence, and effectiveness. J Am Geriatr Soc 2013;61: 483-94. doi:10.1111/ jgs.12169

53 Ranasinghe DC, Shinmoto Torres RL, Hill K, Visvanathan R. Low cost and batteryless sensor-enabled radio frequency identification tag based approaches to identify patient bed entry and exit posture transitions. Gait Posture 2014;39: 118-23. doi:10.1016/j. gaitpost.2013.06.009

(c) BMJ Publishing Group Ltd 2015

Appendix 